\title{
Development and validation of Smartphone based Health Education Module towards the prevention of respiratory tract infection in Malaysian Hajj pilgrims
}

${ }^{1}$ Mohammed, D.G., ${ }^{1}$ Habsah, H., ${ }^{2}$ Naing, N.N., ${ }^{3}$ Rafie, M.A., ${ }^{4}$ Nadiah, W.A., ${ }^{5}$ Arifin, W.N., Aisha, A.B.

${ }^{1}$ Department of Microbiologyand Parasitology, School ofMedical Sciences, Universiti Sains Malaysia; ${ }^{2}$ Faculty of Medicine, Medical Campus, Universiti Sultan Zainal Abidin; ${ }^{3}$ School of Computer Sciences, Universiti Sains Malaysia $;{ }^{4}$ Faculty of Health Sciences, Gong Badak campus, Universiti Sultan Zainal Abidin, Terengganu, Malaysia; ${ }^{5}$ Unit of Biostatistics and Research Methodology, School of Medical Sciences, Universiti Sains Malaysia Health Campus, ${ }^{6}$ Centre for Language Studies and Generic Development, Universiti Malaysia Kelantan, Bachok, Kelantan, Malaysia

\begin{abstract}
Objectives: We aimed to develop and evaluate the efficacy of a health education program for increasing knowledge, changing attitudes and promoting preventive practices to reduce the incidence of RTIs among Malaysian Hajj pilgrims.

Methods: A quasi-experimental study was conducted among 132 Hajj pilgrims attending Hajj orientation programme organized by a private Hajj companies. Hajj pilgrims were sequentially enrolled and assigned to receive smartphone health education application in the intervention group $(\mathrm{n}=66)$ and another different smartphone application on general Hajj process in the control group $(n=66)$. Data were collected from August 2018 to April 2019 at baseline prior to departure and post-intervention, immediately after return from Saudi Arabia using a validated questionnaire.
\end{abstract}

Results: There was no significant difference with respect to socio-demographic characteristics, $\mathrm{KAP}$ of the respondents in the intervention and control group at baseline. However, there was significant improvement in knowledge in the intervention group compared to the control group, based on time $(p=0.005, \grave{\eta} 2=0.075)$ and group and time interaction $(p=0.031, \dot{\eta} 2=0.046)$. Likewise, there was significant improvement in attitude based on time $(p=0.035, \grave{\eta} 2=0.044)$. Similarly, there was significant main effect in practice based on time $(p=<0.001, \grave{\eta} 2=0.155)$ and interaction of group with time $(p=0.042, \grave{\eta} 2=0.041)$.

Keywords: Health education, Hajj, respiratory tract infection 